\title{
A Discrete Variational Integrator for Optimal Control Problems on $\mathrm{SO}(3)$
}

\author{
Islam I. Hussein
}

Melvin Leok

\begin{abstract}
In this paper we study a discrete variational optimal control problem for the rigid body. The cost to be minimized is the external torque applied to move the rigid body from an initial condition to a pre-specified terminal condition. Instead of discretizing the equations of motion, we use the discrete equations obtained from the discrete Lagranged'Alembert principle, a process that better approximates the equations of motion. Within the discrete-time setting, these two approaches are not equivalent in general. The kinematics are discretized using a natural Lie-algebraic formulation that guarantees that the flow remains on the Lie group SO $(3)$ and its algebra $\mathfrak{s o}(3)$. We use Lagrange's method for constrained problems in the calculus of variations to derive the discretetime necessary conditions. We give a numerical example for a three-dimensional rigid body maneuver.
\end{abstract}

\section{INTRODUCTION}

This paper deals with a structure-preserving computational approach to the optimal control problem of minimizing the control effort necessary to perform an attitude transfer from an initial state to a prescribed final state, in the absence of a potential field. The configuration of the rigid body is given by the rotation matrix from the body frame to the spatial frame, which is an element of the group of orientation-preserving isometries in $\mathbb{R}^{3}$. The state of the rigid body is described by the rotation matrix and its angular velocity.

To motivate the computational approach we adopt in the discrete-time case, we first revisit the variational continuous-time optimal control problem. The continuoustime extremal solutions to this optimal control problem have certain special features, since they arise from variational principles. General numerical integration methods, including the popular Runge-Kutta schemes, typically preserve neither first integrals nor the characteristics of the configuration space. Geometric integrators are the class of numerical integration schemes that preserve such properties, and a good survey can be found in [1]. Techniques particular to Hamiltonian systems are also discussed in [2] and [3].

Our approach to discretizing the optimal control problem is in contrast to traditional techniques such as collocation, wherein the continuous equations of motion are imposed as constraints at a set of collocation points. In our approach,

Islam Hussein is Assistant Professor of Mechanical Engineering at Worcester Polytechnic Institute, ihussein@uiuc.edu.

Melvin Leok is Assistant Professor of Mathematics at Purdue University, West Lafayette,mleok@umich.edu.

Amit Sanyal is Post-Doctoral Research Associate at Arizona State University, Tempe, sanyal@asu.edu.

Anthony Bloch is Professor of Mathematics at the University of Michigan, Ann Arbor, abloch@umich.edu.
Amit K. Sanyal

Anthony M. Bloch modeled after [4], the discrete equations of motion are derived from a discrete variational principle, and this induces constraints on the configuration at each discrete time step.

This approach yields discrete dynamics that are more faithful to the continuous equations of motion, and consequently yields more accurate numerical solutions to the optimal control problem. This feature is extremely important in computing accurate (sub)optimal trajectories for longterm spacecraft attitude maneuvers. For example, in [5], the authors propose an imaging spacecraft formation design that requires a continuous attitude maneuver over a period of 77 days in a low Earth orbit. Hence, the attitude maneuver has to be very accurate to meet tight imaging constraints over long time ranges. The proposed variational scheme can also be easily extended to other types of Lie groups. For example, in long range inter-planetary orbit transfers (see, for example, [6]), one is interested in computing optimal or suboptimal trajectories on the group of rigid body motions $\mathrm{SE}(3)$ with a high degree of accuracy. Similar requirements also apply to the control of quantum systems. For example, efficient construction of quantum gates is a problem on the unitary Lie group $\mathrm{SU}(N)$. This is an optimal control problem, where one wishes to steer the identity operator to the desired unitary operator (see, for example, [7] and [8]).

Moreover, an important feature of the way we discretize the optimal control problem is that it is $\mathrm{SO}(3)$-equivariant. This is desirable, since it ensures that our numerical results are independent of the choice of coordinates and coordinate frames. This is in contrast to methods based on coordinatizing the rotation group using quaternions, (modified) Rodrigues parameters, and Euler angles, as given in the survey [9]. Even if the optimal cost function is $\mathrm{SO}(3)$ invariant, as in [10], the use of generalized coordinates imposes constraints on the attitude kinematics.

For the purpose of numerical simulation, the corresponding discrete optimal control problem is posed on the discrete state space as a two stage discrete variational problem. In the first step, we derive the discrete dynamics for the rigid body in the context of discrete variational mechanics [11]. This is achieved by considering the discrete Lagranged'Alembert variational principle [12] in combination with essential ideas from Lie group methods [13], which yields a Lie group variational integrator [14]. This integrator explicitly preserves the Lie group structure of the configuration space, and is similar to the integrators introduced in [15] for a rigid body in an external field, and in [16] for full body dynamics. These discrete equations are then imposed as constraints to be satisfied by the extremal solutions to the 
discrete optimal control problem, and we obtain the discrete extremal solutions in terms of the given terminal states.

The paper is organized as follows. As motivation, in Section II, we study the optimal control problem in continuoustime. In Section III, we study the discrete-time case. In particular, in Section III-A we state the optimal control problem and describe our approach. In Section III-B, we derive the discrete-time equations of motion for the rigid body starting with the discrete Lagrange-d'Alembert principle. These equations are used in Section III-C for the optimal control problem. In Section IV, we describe an algorithm for solving the general nonlinear, implicit necessary conditions for $\mathrm{SO}(3)$ and give numerical examples for rest-to-rest and slew-up spacecraft maneuvers.

\section{Continuous-Time Results}

\section{A. Problem Formulation}

In this paper, the natural pairing between $\mathfrak{s o}^{*}(3)$ and $\mathfrak{s o}(3)$ is denoted by $\langle\cdot, \cdot\rangle$. Let $\ll \cdot, \cdot \gg$ and $\ll \cdot, \cdot\rangle_{*}$ denote the standard (induced by the Killing form) inner product on $\mathfrak{s o}(3)$ and $\mathfrak{s o}^{*}(3)$, respectively. The inner product $\ll \cdot, \cdot \gg_{*}$ is naturally induced from the standard norm $\ll \boldsymbol{\xi}, \boldsymbol{\omega} \gg=-\frac{1}{2} \operatorname{Tr}\left(\boldsymbol{\xi}^{T} \boldsymbol{\omega}\right)$, for all $\boldsymbol{\xi}, \boldsymbol{\omega} \in \mathfrak{s o}(3)$, through

$$
\begin{aligned}
\ll \boldsymbol{\eta}, \boldsymbol{\varphi} \gg_{*} & =\left\langle\boldsymbol{\eta}, \boldsymbol{\varphi}^{\sharp}\right\rangle=\langle\boldsymbol{\eta}, \boldsymbol{\omega}\rangle=\left\langle\boldsymbol{\xi}^{b}, \boldsymbol{\omega}\right\rangle \\
& =\ll \boldsymbol{\xi}, \boldsymbol{\omega} \gg,
\end{aligned}
$$

where $\boldsymbol{\varphi}=\boldsymbol{\omega}^{b} \in \mathfrak{s o}^{*}(3)$ and $\boldsymbol{\eta}=\boldsymbol{\xi}^{b} \in \mathfrak{s o}^{*}(3)$, with $\boldsymbol{\xi}, \boldsymbol{\omega} \in$ $\mathfrak{s o}(3)$ and $b$ and $\sharp$ are the musical isomorphisms (see $\S 2.5$ of [17]) with respect to the standard metric $\ll \cdot, \cdot \gg$. On $\mathfrak{s o}(3)$, these isomorphisms correspond to the transpose operation. That is, we have $\varphi=\omega^{\mathrm{T}}$ and $\boldsymbol{\eta}=\boldsymbol{\xi}^{\mathrm{T}}$.

Let $\mathbf{J}: \mathfrak{s o}(3) \rightarrow \mathfrak{s o}^{*}(3)$ be the positive definite inertia operator. It can be shown that

$$
\langle\mathbf{J}(\boldsymbol{\xi}), \boldsymbol{\omega}\rangle=\langle\mathbf{J}(\boldsymbol{\omega}), \boldsymbol{\xi}\rangle .
$$

On $\mathfrak{s o}(3), \mathbf{J}$ is given by $\mathbf{J}(\boldsymbol{\xi})=J \boldsymbol{\xi}+\boldsymbol{\xi} J$, where $J$ is a positive definite symmetric matrix (see, for example, [17], [18]). Moreover, we also have $\mathbf{J}\left(\boldsymbol{\eta}^{\sharp}\right)^{\sharp}=\left(J \boldsymbol{\eta}^{\mathrm{T}}+\boldsymbol{\eta}^{\mathrm{T}} J\right)^{\mathrm{T}}=$ $\mathbf{J}(\boldsymbol{\eta})$, which is an abuse of notation since $\boldsymbol{\eta} \in \mathfrak{s o}^{*}(3)$. For the sake of generality and mathematical accuracy, we will use the general definitions, though it is helpful to keep the above identifications for $\mathfrak{s o}(3)$ in mind.

In this section we review some continuous-time optimal control results using a simple optimal control example on $\mathrm{SO}(3)$. Here, we minimize the norm squared of the control torque $\tau \in \mathfrak{s o}^{*}(3)$ applied to rotate a rigid body subject to the Lagrange-d'Alembert principle for the rigid body ${ }^{1}$ whose configuration is given by $\mathbf{R} \in \mathbf{S O}(3)$ and body angular velocity is given by $\Omega \in \mathfrak{s o}(3)$. We require that the system evolve from an initial state $\left(\mathbf{R}_{0}, \boldsymbol{\Omega}_{0}\right)$ to a final state $\left(\mathbf{R}_{T}, \boldsymbol{\Omega}_{T}\right)$ at a fixed terminal time $T$. Hence, we have the following minimum control effort optimal control problem.

\footnotetext{
${ }^{1}$ This is equivalent to constraining the problem to satisfy the rigid body equations of motion given by equations (7). However, for the sake of generality that will be appreciated in the discrete-time problem, we choose to treat the Lagrange-d'Alembert principle as the constraint as opposed to the rigid body equations of motion. Both are equivalent in the continuoustime case but are generally not equivalent in the discrete-time case.
}

Problem 2.1: Minimize

subject to

$$
\mathcal{J}=\frac{1}{2} \int_{0}^{T} \ll \tau, \tau \gg_{*} \mathrm{~d} t
$$

1) satisfying Lagrange-d'Alembert principle:

$$
\boldsymbol{\delta} \int_{0}^{T} \frac{1}{2}\langle\mathbf{J}(\boldsymbol{\Omega}), \boldsymbol{\Omega}\rangle \mathrm{d} t+\int_{0}^{T}\langle\boldsymbol{\tau}, \mathbf{W}\rangle \mathrm{d} t=0
$$

subject to $\dot{\mathbf{R}}=\mathbf{R} \boldsymbol{\Omega}$, where $\mathbf{W}$ is the variation vector field to be defined below,

2) and the boundary conditions

$$
\begin{aligned}
\mathbf{R}(0) & =\mathbf{R}_{0}, \boldsymbol{\Omega}(0)=\boldsymbol{\Omega}_{0}, \\
\mathbf{R}(T) & =\mathbf{R}_{T}, \boldsymbol{\Omega}(T)=\boldsymbol{\Omega}_{T} .
\end{aligned}
$$

We now show that this is equivalent to the following problem formulation, where the rigid body equations of motion replace the Lagrange-d'Alembert principle.

Problem 2.2: Minimize

$$
\mathcal{J}=\frac{1}{2} \int_{0}^{T} \ll \boldsymbol{\tau}, \boldsymbol{\tau} \gg_{*} \mathrm{~d} t
$$

subject to

1) the dynamics

$$
\begin{aligned}
\dot{\mathbf{R}} & =\mathbf{R} \boldsymbol{\Omega} \\
\dot{\mathbf{M}} & =\operatorname{ad}_{\mathbf{\Omega}}^{*} \mathbf{M}+\boldsymbol{\tau}=[\mathbf{M}, \boldsymbol{\Omega}]+\boldsymbol{\tau},
\end{aligned}
$$

where $\mathbf{M}=\mathbf{J}(\boldsymbol{\Omega}) \in \mathfrak{s o}^{*}(3)$ is the momentum,

2) and the boundary conditions

$$
\begin{aligned}
\mathbf{R}(0) & =\mathbf{R}_{0}, \boldsymbol{\Omega}(0)=\boldsymbol{\Omega}_{0}, \\
\mathbf{R}(T) & =\mathbf{R}_{T}, \boldsymbol{\Omega}(T)=\boldsymbol{\Omega}_{T} .
\end{aligned}
$$

In the above, ad* is the dual of the adjoint representation, ad, of $\mathfrak{s o}(3)$ and is given by $\operatorname{ad}_{\boldsymbol{\xi}}^{*} \boldsymbol{\eta}=-[\boldsymbol{\xi}, \boldsymbol{\eta}] \in \mathfrak{s o}^{*}(3)$, for all $\boldsymbol{\xi} \in \mathfrak{s o}(3)$ and $\boldsymbol{\eta} \in \mathfrak{s o}^{*}(3)$. Recall that the bracket is defined by $[\boldsymbol{\xi}, \boldsymbol{\omega}]=\boldsymbol{\xi} \boldsymbol{\omega}-\boldsymbol{\omega} \boldsymbol{\xi}$.

\section{B. The Lagrange-d'Alembert Principle and the Rigid Body Equations of Motion}

In this section we derive the forced rigid body equations of motion (equations (7)) from the Lagrange-d'Alembert principle. In dealing with the kinematic constraint, $\dot{\mathbf{R}}=$ $\mathbf{R} \Omega$, we may either append it to the Lagrangian using the method of Lagrange multipliers, or we can directly compute the constrained variations (see $\S 13.5$ of [19]). Here, we take the direct approach as it yields a more concise derivation.

First, we take variations of the kinematic condition $\Omega=$ $\mathbf{R}^{-1} \mathbf{R}$ to obtain $\delta \boldsymbol{\Omega}=-\mathbf{R}^{-1}(\boldsymbol{\delta} \mathbf{R}) \mathbf{R}^{-1} \dot{\mathbf{R}}+\mathbf{R}^{-1} \boldsymbol{\delta} \dot{\mathbf{R}}$. As defined previously, we have $\mathbf{W}=\mathbf{R}^{-1} \boldsymbol{\delta} \mathbf{R}$ and, therefore, $\dot{\mathbf{W}}=-\mathbf{R}^{-1} \dot{\mathbf{R}} \mathbf{R}^{-1} \boldsymbol{\delta} \mathbf{R}+\mathbf{R}^{-1} \boldsymbol{\delta} \dot{\mathbf{R}}=-\boldsymbol{\Omega} \mathbf{W}+\mathbf{R}^{-1} \boldsymbol{\delta} \dot{\mathbf{R}}$, since $\delta \dot{\mathbf{R}}=\frac{\mathrm{d}}{\mathrm{d} t} \boldsymbol{\delta} \mathbf{R}$ (see [20], p. 52). Hence, we have

$$
\delta \boldsymbol{\Omega}=-\mathbf{W} \boldsymbol{\Omega}+\boldsymbol{\Omega} \mathbf{W}+\dot{\mathbf{W}}=\operatorname{ad}_{\Omega} \mathbf{W}+\dot{\mathbf{W}} .
$$

Taking variations of the Lagrange-d'Alembert principle we obtain

$$
\int_{0}^{T}\langle\mathbf{J}(\boldsymbol{\Omega}), \boldsymbol{\delta} \boldsymbol{\Omega}\rangle+\langle\boldsymbol{\tau}, \mathbf{W}\rangle \mathrm{d} t=0
$$

Using the variation in equation (9) and integrating by parts, we obtain $0=\int_{0}^{T}\left\langle-\dot{\mathbf{M}}+\operatorname{ad}_{\Omega}^{*} \mathbf{M}+\boldsymbol{\tau}, \mathbf{W}\right\rangle \mathrm{d} t+[\langle\mathbf{J}(\boldsymbol{\Omega}), \mathbf{W}(t)\rangle]_{0}^{T}$,
where we have used the property

$$
\left\langle\boldsymbol{\eta}, \operatorname{ad}_{\boldsymbol{\omega}} \boldsymbol{\xi}\right\rangle=\left\langle\operatorname{ad}_{\boldsymbol{\omega}}^{*} \boldsymbol{\eta}, \boldsymbol{\xi}\right\rangle, \boldsymbol{\eta} \in \mathfrak{s o}^{*}(3), \boldsymbol{\omega}, \boldsymbol{\xi} \in \mathfrak{s o}(3) .
$$


This gives the desired result, with $\mathbf{M}=\mathbf{J}(\boldsymbol{\Omega})$.

\section{Continuous-Time Variational Optimal Control Problem}

A direct variational approach is used here to derive the necessary conditions for the optimal control Problem (2.2).

A Second Order Direct Approach. "Second order" is used here to reflect the fact that we now study variations of second order dynamical equations as opposed to the kinematic direct approach studied in Section II-B. We now give the resulting necessary conditions using a direct approach as in [19]. We already computed the variations of $\mathbf{R}$ and $\boldsymbol{\Omega}$. These were as follows: $\delta \mathbf{R}=\mathbf{R W}$ and $\delta \boldsymbol{\Omega}=\operatorname{ad}_{\boldsymbol{\Omega}} \mathbf{W}+\dot{\mathbf{W}}$. We now compute the variation of $\dot{\mathbf{M}}$ with the goal of obtaining the proper variations for $\tau$ :

$$
\boldsymbol{\delta} \dot{\mathbf{M}}=\mathbf{J}(\boldsymbol{\delta} \dot{\boldsymbol{\Omega}})=\mathbf{J}\left(\frac{\mathrm{d}}{\mathrm{d} t} \boldsymbol{\delta} \boldsymbol{\Omega}+\mathcal{R}(\mathbf{W}, \boldsymbol{\Omega}) \boldsymbol{\Omega}\right),
$$

where $\mathcal{R}$ is the curvature tensor on $\mathrm{SO}(3)$. The curvature tensor $\mathcal{R}$ arises due to the identity (see [20], page 52)

$$
\frac{\partial}{\partial \epsilon} \frac{\partial}{\partial t} \mathbf{Y}-\frac{\partial}{\partial t} \frac{\partial}{\partial \epsilon} \mathbf{Y}=\mathcal{R}(\mathbf{W}, \mathbf{Y}) \boldsymbol{\Omega}
$$

where $\mathbf{Y} \in \mathrm{TSO}(3)$ is any vector field along the curve $\mathbf{R}(t) \in \mathbf{S O}(3)$. Taking variations of $\dot{\mathbf{M}}=\operatorname{ad}_{\mathbf{\Omega}}^{*} \mathbf{M}+\boldsymbol{\tau}$ we obtain $\delta \dot{M}=\operatorname{ad}_{\delta \Omega}^{*} \mathbf{M}+\operatorname{ad}_{\Omega}^{*} \delta \mathrm{M}+\delta \tau$. We now have the desired variation in $\tau$ :

$$
\begin{aligned}
\delta \boldsymbol{\tau}= & \mathbf{J}(\mathcal{R}(\mathbf{W}, \boldsymbol{\Omega}) \boldsymbol{\Omega})+\frac{\mathrm{d}}{\mathrm{d} t} \mathbf{J}(\boldsymbol{\delta} \boldsymbol{\Omega})-\operatorname{ad}_{\delta \boldsymbol{\Omega}}^{*} \mathbf{M} \\
& -\operatorname{ad}_{\boldsymbol{\Omega}}^{*} \boldsymbol{\delta} \mathbf{M} .
\end{aligned}
$$

Take variations of the cost functional (6) to obtain:

$$
\begin{aligned}
& \boldsymbol{\delta} \mathcal{J}=\int_{0}^{T}\left\langle\mathbf{J}(\ddot{\zeta})-\operatorname{ad}_{\boldsymbol{\Omega}}^{*}(\mathbf{J}(\dot{\varsigma}))+\dot{\boldsymbol{\eta}}-\frac{\mathrm{d}}{\mathrm{d} t}\left(\operatorname{ad}_{\boldsymbol{\varsigma}}^{*} \mathbf{M}\right)\right. \\
& \left.+\left[\mathcal{R}\left(\mathbf{J}(\boldsymbol{\varsigma})^{\sharp}, \boldsymbol{\Omega}\right) \boldsymbol{\Omega}\right]^{b}+\operatorname{ad}_{\boldsymbol{\Omega}}^{*} \operatorname{ad}_{\varsigma}^{*} \mathbf{M}-\operatorname{ad}_{\boldsymbol{\Omega}}^{*} \boldsymbol{\eta}, \mathbf{W}\right\rangle \mathrm{d} t,
\end{aligned}
$$
where $\boldsymbol{\varsigma}=\boldsymbol{\tau}^{\sharp} \in \mathfrak{s o}(3)$ and $\boldsymbol{\eta}=\mathbf{J}\left(\operatorname{ad}_{\boldsymbol{\Omega}} \boldsymbol{\varsigma}\right) \in \mathfrak{s o}^{*}(3)$. Here, we used integration by parts and the boundary conditions (7), equations (9) and (11), and the identities (1), (2) and (10). Hence, we have the following theorem.

Theorem 2.1: The necessary optimality conditions for the problem of minimizing (6) subject to the dynamics (7) and the boundary conditions (8) are given by the single fourth $\operatorname{order}^{2}$ differential equation

$$
\begin{aligned}
0= & \mathbf{J}(\ddot{\varsigma})-\operatorname{ad}_{\boldsymbol{\Omega}}^{*}(\mathbf{J}(\dot{\varsigma}))+\dot{\boldsymbol{\eta}}-\frac{\mathrm{d}}{\mathrm{d} t}\left(\operatorname{ad}_{\boldsymbol{\varsigma}}^{*} \mathbf{M}\right) \\
& +\left[\mathcal{R}\left((\mathbf{J}(\boldsymbol{\varsigma}))^{\sharp}, \boldsymbol{\Omega}\right) \boldsymbol{\Omega}\right]^{b}+\operatorname{ad}_{\boldsymbol{\Omega}}^{*}\left(\operatorname{ad}_{\boldsymbol{\varsigma}}^{*} \mathbf{M}\right)-\operatorname{ad}_{\boldsymbol{\Omega}}^{*} \boldsymbol{\eta},
\end{aligned}
$$

as well as the equations (7) and the boundary conditions (8), where $\varsigma$ and $\boldsymbol{\eta}$ are as defined above.

To obtain above result we used the initial conditions (8), and the fact that the vector fields $\boldsymbol{\Omega}$ and $\mathbf{W}$ are left-invariant vector fields. The curvature tensor is evaluated at a point $\mathbf{R}(t) \neq \mathbf{I}$. That is, we get $\mathcal{R}_{\mathbf{R}_{\epsilon}}\left(\frac{\partial \mathbf{R}_{\epsilon}}{\partial \epsilon}, \frac{\partial \mathbf{R}_{\epsilon}}{\partial t}\right) \boldsymbol{\Omega}$. Evaluating this at $\epsilon=0$ we get: $\mathcal{R}_{\mathbf{R}}(\mathbf{R W}, \mathbf{R} \boldsymbol{\Omega}) \boldsymbol{\Omega}$. Since $\mathbf{R W}$ and $\mathbf{R} \boldsymbol{\Omega}$ are left-invariant vector fields at the group element $\mathbf{R}(t)$, by the identification $\mathrm{T}_{\mathbf{R}} \mathrm{SO}(3) \simeq \mathfrak{s o}(3)$, we have $\mathcal{R}_{\mathbf{R}}(\mathbf{R W}, \mathbf{R} \boldsymbol{\Omega}) \boldsymbol{\Omega}=\mathcal{R}(\mathbf{W}, \boldsymbol{\Omega}) \boldsymbol{\Omega}$, which is the curvature tensor evaluated at the identity element. For a compact semi-simple Lie group $\mathrm{G}$ with Lie algebra $\mathfrak{g}$, the curvature

\footnotetext{
${ }^{2}$ Second order in $\boldsymbol{\tau}$ and fourth order in $\mathbf{R}$.
}

tensor, with respect to a bi-invariant metric, is (see [20]):

for all $\mathbf{X}, \mathbf{Y}, \mathbf{Z} \in \mathfrak{g}$.

$$
\mathcal{R}(\mathbf{X}, \mathbf{Y}) \mathbf{Z}=\frac{1}{4} \operatorname{ad}_{\mathrm{ad}_{\mathbf{X}} \mathbf{Y}} \mathbf{Z},
$$

Using a Lagrange multiplier approach, we obtain instead the following theorem.

Theorem 2.2: The necessary optimality conditions for the problem of minimizing (6) subject to the dynamics (7) and the boundary conditions (8) are given by

$$
\begin{aligned}
\boldsymbol{\tau} & =\boldsymbol{\Lambda}_{2} \\
\dot{\boldsymbol{\Lambda}}_{1} & =\left[\mathcal{R}\left(\mathbf{J}\left(\boldsymbol{\Lambda}_{2}\right)^{\sharp}, \boldsymbol{\Omega}\right) \boldsymbol{\Omega}\right]^{b}+\operatorname{ad}_{\boldsymbol{\Omega}}^{*} \boldsymbol{\Lambda}_{1} \\
\dot{\boldsymbol{\Lambda}}_{2} & =-\mathbf{J}^{-1}\left(\boldsymbol{\Lambda}_{1}\right)-\operatorname{ad}_{\boldsymbol{\Omega}} \boldsymbol{\Lambda}_{2}+\mathbf{J}^{-1}\left(\operatorname{ad}_{\boldsymbol{\Lambda}_{2}}^{*} \mathbf{M}\right)
\end{aligned}
$$

and the equations (7) and the boundary conditions (8). The Lagrange multipliers $\boldsymbol{\Lambda}_{1} \in \mathfrak{s o}^{*}(3), \boldsymbol{\Lambda}_{2} \in \mathfrak{s o}(3)$ correspond to the kinematic and dynamics constraints (7), respectively.

Remark 2.1: Note that the equations of motion that arise from the Lagrange-d'Alembert principle are used to define the dynamic constraints. In effect, we minimize $\mathcal{J}$ subject to satisfying the Lagrange-d'Alembert principle. Analogously, the discrete version of the Lagrange-d'Alembert principle will be used to derive the discrete equations of motion in the discrete optimal control problem to be studied in Section III-C. This view is in line with the approach in [4] in that we do not discretize the equations of motion directly, but, instead, we discretize the Lagrange-d'Alembert principle. These two approaches are not equivalent in general.

Corollary 2.1: The necessary optimality conditions of Theorem 2.1 are equivalent to those of Theorem 2.2.

Proof. In Theorem 2.2, differentiate $\boldsymbol{\Lambda}_{2}$ once and then use all three differential equations to replace $\boldsymbol{\Lambda}_{1}$ and $\boldsymbol{\Lambda}_{2}$ with expressions involving only $\tau, \mathbf{M}$ and $\boldsymbol{\Omega}$.

\section{Discrete-Time Results}

\section{A. Problem Formulation}

In this section we give the discrete version of the problem introduced in Section II-A. So, we consider minimizing the norm squared of the control torque $\tau$ subject to satisfaction of the discrete Lagrange-d'Alembert principle for the rigid body whose configuration and body angular velocity at time step $t_{k}$ are given by $\mathbf{R}_{k} \in \mathbf{S O}(3)$ and $\Omega_{k} \in \mathfrak{s o}(3)$, respectively. The kinematic constraint may be expressed as

$$
\mathbf{R}_{k+1}=\mathbf{R}_{k} \exp \left(h \boldsymbol{\Omega}_{k}\right)=\mathbf{R}_{k} \mathbf{g}_{k},
$$

where $h$ is the integration time step, $\exp : \mathfrak{s o}(3) \rightarrow \mathbf{S O}(3)$ is the exponential map and $\mathbf{g}_{k}=\exp \left(h \boldsymbol{\Omega}_{k}\right)$. The boundary conditions are given by $\left(\mathbf{R}_{0}^{*}, \boldsymbol{\Omega}_{0}^{*}\right)$ and $\left(\mathbf{R}_{N}^{*}, \boldsymbol{\Omega}_{N-1}^{*}\right)$, where $t_{0}=0$ and $N=T / h$ is such that $t_{N}=T$.

The reason we constrain $\Omega$ at $t=h(N-1)$ instead of at $t=h N$ is that a constraint on $\boldsymbol{\Omega}_{k} \in \mathfrak{s o}(3)$ corresponds, by left translations to a constraint on $\dot{\mathbf{R}}_{k} \in \mathrm{T}_{\mathbf{R}_{k}} \mathbf{S O}(3)$. In turn, in the discrete setting and depending on the choice of discretization, this corresponds to a constraint on the neighboring discrete points. With our choice of discretization (equation (14)), this corresponds to constraints on $\mathbf{R}_{k}$ and $\mathbf{R}_{k+1}$. Hence, to ensure that the effect of the terminal constraint on $\Omega$ is correctly accounted for, the constraint 
must be imposed on $\boldsymbol{\Omega}_{N-1}$, which entails some constraints on variations at both $\mathbf{R}_{N-1}$ and $\mathbf{R}_{N}$. We will return to this point later in the paper.

Equation (14) is just one way of discretizing the kinematics of the rigid body. In the case of planar rigid body dynamics, this leads to the first-order Euler approximation. However, on $\mathrm{SO}(3)$, our approach yields a novel discretization. We make the above choice for discretization as it guarantees, in general, that the angular velocity matrix $\boldsymbol{\Omega}_{k}$ remains on the algebra $\mathfrak{s o}(3)$ by using the exponential map. This is natural to do in the context of discrete variational numerical solvers (for both initial value and two point boundary value problems). Following the methodology of [4], we have the following optimal control problem.

Problem 3.1: Minimize

$$
\mathcal{J}=\sum_{k=0}^{N} \frac{1}{2} \ll \boldsymbol{\tau}_{k}, \boldsymbol{\tau}_{k} \gg_{*}
$$

subject to

1) satisfying the discrete Lagrange-d'Alembert principle:

$$
\boldsymbol{\delta} \sum_{k=0}^{N-1} \frac{1}{2}\left\langle\mathbf{J}\left(\boldsymbol{\Omega}_{k}\right), \boldsymbol{\Omega}_{k}\right\rangle+\sum_{k=0}^{N}\left\langle\boldsymbol{\tau}_{k}, \mathbf{W}_{k}\right\rangle=0,
$$

subject to $\mathbf{R}_{0}=\mathbf{R}_{0}^{*}, \mathbf{R}_{N}=\mathbf{R}_{N}^{*}$ and $\mathbf{R}_{k+1}=\mathbf{R}_{k} \mathbf{g}_{k}$, $k=0,1, \ldots, N-1$, where $\mathbf{W}_{k}$ is the variation vector field at time step $t_{k}$ satisfying $\delta \mathbf{R}_{k}=\mathbf{R}_{k} \mathbf{W}_{k}$,

2) and the boundary conditions

$$
\begin{aligned}
\mathbf{R}_{0} & =\mathbf{R}_{0}^{*}, \boldsymbol{\Omega}_{0}=\boldsymbol{\Omega}_{0}^{*}, \\
\mathbf{R}_{N} & =\mathbf{R}_{N}^{*}, \boldsymbol{\Omega}_{N-1}=\boldsymbol{\Omega}_{N-1}^{*} .
\end{aligned}
$$

The following formulation is equivalent, where the discrete rigid body equations of motion replace the Lagranged'Alembert principle constraint.

Problem 3.2: Minimize

$$
\mathcal{J}=\sum_{k=0}^{N} \frac{1}{2} \ll \boldsymbol{\tau}_{k}, \boldsymbol{\tau}_{k} \gg_{*}
$$

subject to

1) the discrete dynamics

$$
\begin{aligned}
& \mathbf{R}_{k+1}=\mathbf{R}_{k} \mathbf{g}_{k}, k=0, \ldots, N-1 \\
& \mathbf{M}_{k}=\operatorname{Ad}_{\mathbf{g}_{k}}^{*}\left(h \boldsymbol{\tau}_{k}+\mathbf{M}_{k-1}\right), k=1, \ldots, N-1, \\
& \mathbf{M}_{k}=\mathbf{J}\left(\boldsymbol{\Omega}_{k}\right), k=0, \ldots, N-1,
\end{aligned}
$$

2) and the boundary conditions

$$
\begin{aligned}
\mathbf{R}_{0} & =\mathbf{R}_{0}^{*}, \boldsymbol{\Omega}_{0}=\boldsymbol{\Omega}_{0}^{*}, \\
\mathbf{R}_{N} & =\mathbf{R}_{N}^{*}, \boldsymbol{\Omega}_{N-1}=\boldsymbol{\Omega}_{N-1}^{*} .
\end{aligned}
$$

Regarding terminal velocity conditions, note that in the second of equations (19) if we let $k=N$ we find that $\boldsymbol{\Omega}_{N}$ appears in the equation. A constraint on $\boldsymbol{\Omega}_{N}$ dictates constraints at the points $\mathbf{R}_{N}$ and $\mathbf{R}_{N+1}$ through the first equation in (19). Since we only consider time points up to $t=N h$, we can not allow $k=N$ in the second of equations (19) and hence our terminal velocity constraints are posed in terms of $\boldsymbol{\Omega}_{N-1}$ instead of $\boldsymbol{\Omega}_{N}$.

As mentioned above, $\mathbf{W}_{k}$ is a variation vector field associated with the perturbed group element $\mathbf{R}_{k}^{\epsilon}$. Likewise, we need to define a variation vector field associated with the element $\mathbf{g}_{k}=\exp \left(h \boldsymbol{\Omega}_{k}\right)$. First, let the perturbed variable $\mathbf{g}_{k}^{\epsilon}$ be defined by

$$
\mathbf{g}_{k}^{\epsilon}=\mathbf{g}_{k} \exp \left(\epsilon h \boldsymbol{\delta} \boldsymbol{\Omega}_{k}\right),
$$

where

$$
\boldsymbol{\delta} \boldsymbol{\Omega}=\left.\frac{\partial \boldsymbol{\Omega}_{k}^{\epsilon}}{\partial \epsilon}\right|_{\epsilon=0} .
$$

Note that $\left.\mathbf{g}_{k}^{\epsilon}\right|_{\epsilon=0}=\mathbf{g}_{k}$ as desired. Moreover, we have

$$
\boldsymbol{\delta} \mathbf{g}_{k}=\left.\mathbf{g}_{k}\left(h \boldsymbol{\delta} \Omega_{k}\right) \exp \left(\epsilon h \delta \Omega_{k}\right)\right|_{\epsilon=0}=h \mathbf{g}_{k} \boldsymbol{\delta} \boldsymbol{\Omega}_{k} .
$$

This will be needed later when taking variations.

\section{B. The Discrete Lagrange-d'Alembert Principle and the Rigid Body Equations of Motion}

In this section we derive the discrete forced rigid body equations of motion (equations (19)) starting with the discrete Lagrange-d'Alembert principle. As in the continuous case, we will compute the constrained variation of $\boldsymbol{\delta} \boldsymbol{\Omega}_{k}$. We begin by rewriting the kinematic constraint as $\exp ^{-1}\left(\mathbf{R}_{k}^{-1} \mathbf{R}_{k+1}\right)=h \boldsymbol{\Omega}_{k}$, which is easier to handle as an expression over the Lie algebra. Take variations to obtain, $-\mathbf{R}_{k}^{-1}\left(\boldsymbol{\delta} \mathbf{R}_{k}\right) \mathbf{R}_{k}^{-1} \mathbf{R}_{k+1}+\mathbf{R}_{k}^{-1} \boldsymbol{\delta} \mathbf{R}_{k+1}=h \mathbf{g}_{k} \cdot \boldsymbol{\delta} \boldsymbol{\Omega}_{k}$, which is equivalent to $-\mathbf{W}_{k} \mathbf{g}_{k}+\mathbf{g}_{k} \mathbf{W}_{k+1}=h \mathbf{g}_{k} \boldsymbol{\delta} \boldsymbol{\Omega}_{k}$, or

$$
\boldsymbol{\delta} \boldsymbol{\Omega}_{k}=\frac{1}{h}\left[-\mathrm{Ad}_{\mathbf{g}_{k}^{-1}} \mathbf{W}_{k}+\mathbf{W}_{k+1}\right] \text {. }
$$

Note that this is an expression over the Lie algebra $\mathfrak{s o}(3)$.

Taking direct variations of the cost functional we obtain

$$
\begin{aligned}
0 & =\left\langle\boldsymbol{\tau}_{0}-\frac{1}{h} \operatorname{Ad}_{\mathbf{g}_{0}^{-1}}^{*} \mathbf{J}\left(\boldsymbol{\Omega}_{0}\right), \mathbf{W}_{0}\right\rangle \\
& +\left\langle\boldsymbol{\tau}_{N}+\frac{1}{h} \mathbf{J}\left(\Omega_{N-1}\right), \mathbf{W}_{N}\right\rangle \\
& +\sum_{k=1}^{N-1}\left\langle\boldsymbol{\tau}_{k}-\frac{1}{h} \operatorname{Ad}_{\mathbf{g}_{k}^{-1}}^{*} \mathbf{J}\left(\boldsymbol{\Omega}_{k}\right)+\frac{1}{h} \mathbf{J}\left(\boldsymbol{\Omega}_{k-1}\right), \mathbf{W}_{k}\right\rangle .
\end{aligned}
$$

where we have used equation (23). By the boundary conditions $\mathbf{R}_{0}=\mathbf{R}_{0}^{*}$ and $\mathbf{R}_{N}=\mathbf{R}_{N}^{*}$, we have $\mathbf{W}_{0}=0$ and $\mathbf{W}_{N}=0$. Since $\mathbf{W}_{k}, k=1, \ldots, N-1$, are arbitrary and independent, we obtain the equivalent of equations (19).

\section{Discrete-Time Variational Optimal Control Problem}

Analogous to the direct approach in continuous time, here we derive the necessary optimality conditions in a form that does not involve the use of Lagrange multipliers. Using equation (23) and taking variation of the second of equations (19), we obtain

$$
\begin{aligned}
\boldsymbol{\delta} \boldsymbol{\tau}_{k}= & \operatorname{Ad}_{\mathbf{g}_{k}^{-1}}^{*}\left(\frac{1}{h^{2}} \mathbf{J}\left(\mathbf{W}_{k+1}-\operatorname{Ad}_{\mathbf{g}_{k}^{-1}} \mathbf{W}_{k}\right)\right. \\
& \left.+\frac{1}{h}\left[\mathbf{W}_{k+1}-\operatorname{Ad}_{\mathbf{g}_{k}^{-1}} \mathbf{W}_{k}, \mathbf{J}\left(\boldsymbol{\Omega}_{k}\right)\right]\right) \\
& -\frac{1}{h^{2}} \mathbf{J}\left(\mathbf{W}_{k}-\operatorname{Ad}_{\mathbf{g}_{k-1}^{-1}} \mathbf{W}_{k-1}\right),
\end{aligned}
$$

for $k=1, \ldots, N-1$. Taking variations of the cost functional (18) and using equation (24), one obtains after a tedious but straightforward computation an expression for $\boldsymbol{\delta} \mathcal{J}$ in terms of $\delta \boldsymbol{\tau}_{k}$, which we omit because of space restrictions. When $\delta \mathcal{J}$ is equated to zero, the resulting equation gives (boundary) conditions on $\boldsymbol{\tau}_{0}, \boldsymbol{\tau}_{1}, \boldsymbol{\tau}_{N-1}, \boldsymbol{\tau}_{N}$ as well as discrete evolution equations that are written in 
algebraic nonlinear form as:

$$
\begin{aligned}
0= & -\frac{1}{h^{2}}\left(\mathbf{J}\left(\boldsymbol{\tau}_{k}^{\sharp}\right)-\operatorname{Ad}_{\mathbf{g}_{k}^{-1}}^{*} \mathbf{J}\left(\boldsymbol{\tau}_{k+1}^{\sharp}\right)\right. \\
& \left.-\mathbf{J}\left(\operatorname{Ad}_{\mathbf{g}_{k-1}^{-1}} \boldsymbol{\tau}_{k-1}^{\sharp}\right)+\operatorname{Ad}_{\mathbf{g}_{k}^{-1}}^{*} \mathbf{J}\left(\operatorname{Ad}_{\mathbf{g}_{k}^{-1}} \boldsymbol{\tau}_{k}^{\sharp}\right)\right) \\
& -\frac{1}{h}\left(\operatorname{Ad}_{\mathbf{g}_{k}^{-1}}^{*}\left[\mathbf{J}\left(\boldsymbol{\Omega}_{k}\right), \operatorname{Ad}_{\mathbf{g}_{k}^{-1}}\left(\boldsymbol{\tau}_{k}^{\sharp}\right)\right]\right. \\
& \left.-\frac{1}{h}\left[\mathbf{J}\left(\boldsymbol{\Omega}_{k-1}\right), \operatorname{Ad}_{\mathbf{g}_{k-1}^{-1}}\left(\boldsymbol{\tau}_{k-1}^{\sharp}\right)\right]\right)
\end{aligned}
$$

for $k=2, \ldots, N-2$.

A Lagrange multiplier approach yields the following equivalent theorem.

Theorem 3.1: The necessary optimality conditions for the discrete Problem 3.2 are

$$
\begin{aligned}
\mathbf{R}_{k+1}= & \mathbf{R}_{k} \mathbf{g}_{k}, k=1, \ldots, N-2 \\
\mathbf{M}_{k}= & \operatorname{Ad}_{\mathbf{g}_{k}}^{*}\left(h \boldsymbol{\tau}_{k}+\mathbf{M}_{k-1}\right), k=1, \ldots, N-1 \\
0= & \boldsymbol{\Lambda}_{k-1}^{1}-\operatorname{Ad}_{\mathbf{g}_{k}^{-1}}^{*} \boldsymbol{\Lambda}_{k}^{1}, k=2, \ldots, N-2 \\
0= & -\boldsymbol{\Lambda}_{k}^{1}+\mathbf{J}\left(\boldsymbol{\Lambda}_{k}^{2}\right)-\mathbf{J}\left(\operatorname{Ad}_{\mathbf{g}_{k+1}} \boldsymbol{\Lambda}_{k+1}^{2}\right) \\
& +h\left[\mathbf{M}_{k}, \boldsymbol{\Lambda}_{k}^{2}\right], k=1, \ldots, N-2 \\
\boldsymbol{\tau}_{k}= & h\left(\operatorname{Ad}_{\mathbf{g}_{k}} \boldsymbol{\Lambda}_{k}^{2}\right)^{b}, k=1, \ldots, N-1 \\
\mathbf{M}_{k}= & \mathbf{J}\left(\boldsymbol{\Omega}_{k}\right), k=0, \ldots, N-1,
\end{aligned}
$$

and the boundary conditions

$$
\begin{aligned}
\mathbf{R}_{0} & =\mathbf{R}_{0}^{*}, \mathbf{R}_{1}=\mathbf{R}_{0}^{*} \mathbf{g}_{0}^{*}, \boldsymbol{\Omega}_{0}=\boldsymbol{\Omega}_{0}^{*} \\
\mathbf{R}_{N} & =\mathbf{R}_{N}^{*}, \mathbf{R}_{N-1}=\mathbf{R}_{N}^{*}\left(\mathbf{g}_{N-1}^{*}\right)^{-1}, \boldsymbol{\Omega}_{N-1}=\boldsymbol{\Omega}_{N-1}^{*} \\
\boldsymbol{\tau}_{0} & =\boldsymbol{\tau}_{N}=0,
\end{aligned}
$$

where $\mathbf{g}_{0}^{*}=\exp \left(h \boldsymbol{\Omega}_{0}^{*}\right)$ and $\mathbf{g}_{N-1}^{*}=\exp \left(h \boldsymbol{\Omega}_{N-1}^{*}\right)$.

\section{NumericAl APPROACH AND RESUlts}

The first-order optimality equations, equation (25), in combination with the boundary conditions,

$\mathbf{R}_{0}=\mathbf{R}_{0}^{*}, \quad \mathbf{R}_{N}=\mathbf{R}_{N}^{*}, \quad \boldsymbol{\Omega}_{0}=\boldsymbol{\Omega}_{0}^{*}$, and $\boldsymbol{\Omega}_{N-1}=\boldsymbol{\Omega}_{N-1}^{*}$, leave the torques $\boldsymbol{\tau}_{1}, \ldots, \boldsymbol{\tau}_{N-1}$, and the angular velocities $\boldsymbol{\Omega}_{1}, \ldots, \boldsymbol{\Omega}_{N-2}$ as unknowns. By substituting the relations $\mathbf{g}_{k}=\exp \left(h \boldsymbol{\Omega}_{k}\right), \mathbf{M}_{k}=\mathbf{J}\left(\boldsymbol{\Omega}_{k}\right)$, we can rewrite the necessary conditions (25) as follows,

$$
\begin{aligned}
0=-\frac{1}{h^{2}}\left(\mathbf{J}\left(\boldsymbol{\tau}_{k}^{\sharp}\right)-\operatorname{Ad}_{\exp \left(-h \boldsymbol{\Omega}_{k}\right)}^{*} \mathbf{J}\left(\boldsymbol{\tau}_{k+1}^{\sharp}\right)\right. & \\
- & \mathbf{J}\left(\operatorname{Ad}_{\exp \left(-h \boldsymbol{\Omega}_{k-1}\right)} \boldsymbol{\tau}_{k-1}^{\sharp}\right. \\
& \left.+\operatorname{Ad}_{\exp \left(-h \boldsymbol{\Omega}_{k}\right)}^{*} \mathbf{J}\left(\operatorname{Ad}_{\exp \left(-h \boldsymbol{\Omega}_{k}\right)} \boldsymbol{\tau}_{k}^{\sharp}\right)\right) \\
- & \frac{1}{h}\left(\operatorname{Ad}_{\exp \left(-h \boldsymbol{\Omega}_{k}\right)}^{*}\left[\mathbf{J}\left(\boldsymbol{\Omega}_{k}\right), \operatorname{Ad}_{\exp \left(-h \boldsymbol{\Omega}_{k}\right)}\left(\boldsymbol{\tau}_{k}^{\sharp}\right)\right]\right. \\
& \left.-\frac{1}{h}\left[\mathbf{J}\left(\boldsymbol{\Omega}_{k-1}\right), \operatorname{Ad}_{\exp \left(-h \boldsymbol{\Omega}_{k-1}\right)}\left(\boldsymbol{\tau}_{k-1}^{\sharp}\right)\right]\right),
\end{aligned}
$$

Lie algebra isomorphism between $\mathbb{R}^{3}$ and $\mathfrak{s o}(3)$ given by,

$$
\mathbf{v}=\left(v_{1}, v_{2}, v_{3}\right) \mapsto \hat{\mathbf{v}}=\left[\begin{array}{ccc}
0 & -v_{3} & v_{2} \\
v_{3} & 0 & -v_{1} \\
-v_{2} & v_{1} & 0
\end{array}\right],
$$

which maps 3 -vectors to $3 \times 3$ skew-symmetric matrices. In particular, we have the following identities,

$$
[\hat{\mathbf{u}}, \hat{\mathbf{v}}]=(\mathbf{u} \times \mathbf{v})^{\wedge}, \quad \operatorname{Ad}_{\mathbf{A}} \hat{\mathbf{v}}=(\mathbf{A v})^{\wedge} .
$$

Furthermore, we identify $\mathfrak{s o}(3)^{*}$ with $\mathbb{R}^{3}$ by the usual dot product, that is to say if $\boldsymbol{\Pi}, \mathbf{v} \in \mathbb{R}^{3}$, then $\langle\boldsymbol{\Pi}, \hat{\mathbf{v}}\rangle=\boldsymbol{\Pi} \cdot \mathbf{v}$. With this identification, we have that $\operatorname{Ad}_{\mathbf{A}^{-1}}^{*} \boldsymbol{\Pi}=\mathbf{A} \Pi$. Using the identities above, we write the necessary conditions using matrix-vector products and cross products. Then, each of the equations can be interpreted as 3-vector valued functions, and the system of equations can be considered as a $3(2 N-3)$-vector valued function, which is precisely the dimensionality of the unknowns. This reduces the discrete optimal problem to a nonlinear root finding problem.

We used a Newton-Armijo method, a line search algorithm that uses the Newton search direction, and backtracking to ensure sufficient descent of the residual error. The Jacobian is constructed column by column, where the $k$-th column is computed using the following approximation,

$$
\frac{\partial \mathbf{F}}{\partial x_{k}}(\mathbf{x})=\frac{1}{\epsilon} \operatorname{Im}\left[\mathbf{F}\left(\mathbf{x}+i \epsilon \mathbf{e}_{k}\right)\right] \text {, }
$$

where $i=\sqrt{-1}, \mathbf{e}_{k}$ is a basis vector in the $x_{k}$ direction, and $\epsilon$ is of the order of machine epsilon. This method is more accurate than a finite-difference approximation as it does not suffer from round-off errors.

In our numerical simulation, we computed an optimal trajectory for a rest-to-rest maneuver, as illustrated in Figure (1). In this simulation $N=128$, and essentially identical results were obtained for $N=64$. It is worth noting that the results are not rotationally symmetric about the midpoint of the simulation interval, which is due to the fact that our choice of update, $\mathbf{R}_{k+1}=\mathbf{R}_{k} \exp \left(h \boldsymbol{\Omega}_{k}\right)$, does not exhibit time-reversal symmetry. In a forthcoming publication, we will introduce a reversible algorithm to address this issue. 

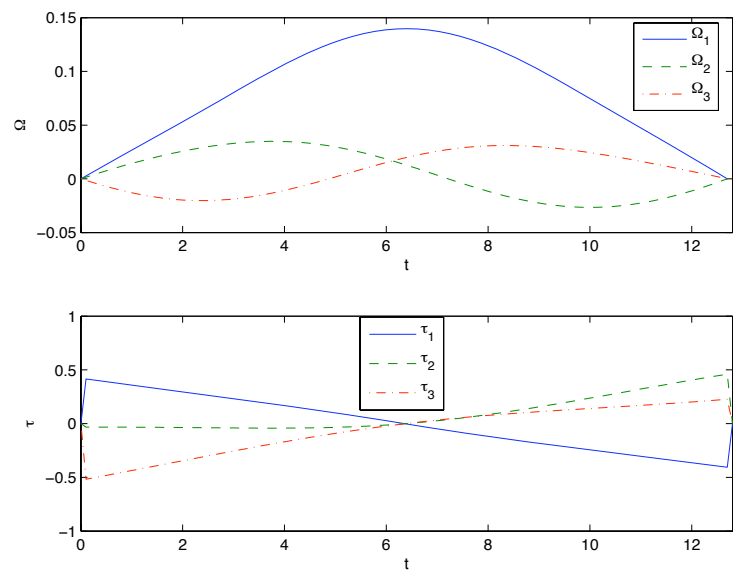

Fig. 1. Discrete optimal rest-to-rest maneuver in $\mathrm{SO}(3)$.
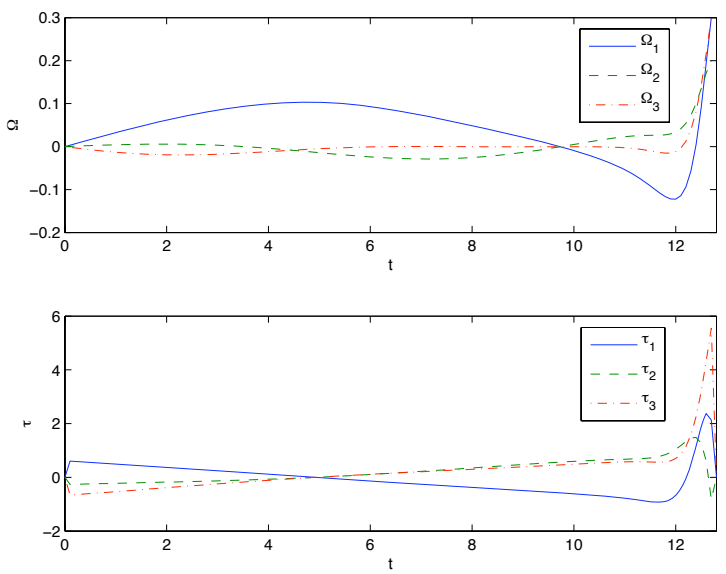

Fig. 2. Discrete optimal slew-up maneuver in $\mathrm{SO}(3)$.

We also present results for an optimal slew-up maneuver in $T=12.8$ seconds from zero initial angular velocity to a final angular velocity of $\Omega_{T}=\left[\begin{array}{llll}0.3 & 0.2 & 0.3\end{array}\right]^{\mathrm{T}}$, illustrated in Figure (2). The resolution is $N=128$, and essentially identical results were obtained for $N=64$.

\section{CONCLUSION}

In this paper we studied the continuous- and discretetime optimal control problem for the rigid body, where the cost to be minimized is the external torque applied to move the rigid body from an initial condition to some pre-specified terminal condition. In the discrete setting, we use the discrete Lagrange-d'Alembert principle to obtain the discrete equations of motion. The kinematics were discretized to guarantee that the flow in phase space remains on the Lie group $\mathrm{SO}(3)$ and its algebra $\mathfrak{s o}(3)$. We described how the necessary conditions can be solved for the general three-dimensional case and gave a numerical example for a three-dimensional rigid body maneuver.

Currently, we are investigating the connections with Pontryagin's maximum principle in continuous- and discretetime. Additionally, we wish to generalize the result to general Lie groups with applications other than rigid body motion on $\mathrm{SO}(3)$. In particular, we are interested in control- ling the motion of a rigid body in space, which corresponds to motion on the non-compact Lie group $\mathrm{SE}(3)$.

\section{ACKNOWLEDGMENTS}

The research of Melvin Leok was partially supported by NSF grant DMS-0504747 and a University of Michigan Rackham faculty grant. The research of Anthony Bloch was supported by NSF grants DMS-0305837, CMS-0408542, and DMS-0604307.

\section{REFERENCES}

[1] E. Hairer, C. Lubich, and G. Wanner, Geometric Numerical Integration. Berlin: Springer, 2002.

[2] B. Leimkuhler and S. Reich, Simulating Hamiltonian Dynamics, ser. Cambridge Monographs on Applied and Computational Mathematics. Cambridge: Cambridge University Press, 2004, vol. 14.

[3] J. M. Sanz-Serna and M. P. Calvo, Numerical Hamiltonian Problems, ser. Applied Mathematics and Mathematical Computation. London: Chapman and Hall, 1994, vol. 7.

[4] O. Junge, J. E. Marsden, and S. Ober-Blöbaum, "Discrete mechanics and optimal control," IFAC Congress, Praha, 2005.

[5] I. I. Hussein, D. J. Scheeres, and D. C. Hyland, "Interferometric observatories in Earth orbit," Journal of Guidance, Control and Dynamics, vol. 27, no. 2, pp. 297-301, 2004.

[6] "New horizons: Pluto-kuiper belt mission," Website, March 2006. [Online]. Available: http://www.nasa.gov/mission_pages/ newhorizons/main/index.html

[7] J. P. Palao and R. Kosloff, "Quantum computing by an optimal control algorithm for unitary transformations," Physical Review Letters, vol. 89, p. 188301, 2002.

[8] N. Khaneja, S. J. Glaser, and R. W. Brockett, "Sub-Riemannian geometry and optimal control of three spin systems," Physical Review $A$, vol. 65 , p. $032301,2002$.

[9] S. L. Scrivener and R. C. Thompson, "Survey of time-optimal attitude maneuvers," Journal of Guidance, Control, and Dynamics, vol. 17, no. 2, pp. 225-233, 1994.

[10] H. Schaub, J. L. Junkins, and R. D. Robinett, "New attitude penalty functions for spacecraft optimal control problems," AIAA Guidance, Navigation, and Control Conference, 1996.

[11] J. Marsden and M. West, "Discrete mechanics and variational integrators," Acta Numerica, vol. 10, pp. 357-514, 2001.

[12] C. Kane, J. E. Marsden, M. Ortiz, and M. West, "Variational integrators and the newmark algorithm for conservative and dissipative mechanical systems," International Journal of Numerical Methods in Engineering, vol. 49, no. 10, pp. 1295-1325, 2000.

[13] A. Iserles, H. Munthe-Kaas, S. P. N. rsett, and A. Zanna, "Lie group methods," Acta Numerica, vol. 9, pp. 215-265, 2000.

[14] M. Leok, "Generalized galerkin variational integrators," 2004, preprint, arXiv:math.NA/0508360.

[15] T. Lee, M. Leok, and N. H. McClamroch, "A Lie group variational integrator for the attitude dynamics of a rigid body with applications to the 3D pendulum," Proceedings of the IEEE Conference on Control Applications, pp. 962-967, 2005.

[16] - -, "Lie group variational integrators for the full body problem," Computer Methods in Applied Mechanics and Engineering, 2005, submitted, arXiv:math.NA/0508365.

[17] A. Bloch, J. Baillieul, P. E. Crouch, and J. E. Marsden, Nonholonomic Mechanics and Control. New York, NY: Springer-Verlag, 2003.

[18] I. I. Hussein and A. M. Bloch, "Optimal trajectory tracking on the group of rigid body motions," 2005 IEEE Conference on Decision and Control, 2005.

[19] J. E. Marsden and T. S. Ratiu, Introduction to Mechanics and Symmetry. New York, NY: Springer-Verlag, 1999.

[20] J. Milnor, Morse Theory. Princeton, NJ: Princeton University Press, 1963. 